\title{
Erratum: Double-time correlation functions of two quantum operations in open systems [Phys. Rev. A 96, 042111 (2017)]
}

\author{
Masashi Ban
}

(Q) (Received 24 May 2018; published 11 June 2018)

DOI: 10.1103/PhysRevA.97.069901

The original paper contains several typographical errors which are as follows: (1) On the right-hand side of Eqs. (40), (43), (44), and (46) and on the left-hand side of Eqs. (58)-(60), $\{\cdots, \cdots\}$ should be replaced by $[\cdots, \cdots]$. (2) In Eqs. (A3) and (A5), the Liouvillian superoperator $\mathcal{L}(t)$ should be replaced by $\mathcal{L}_{0}(t)$. (3) Eq. (A14) should read $\hat{\mathcal{J}}_{S}(t \mid g)=\operatorname{Tr}_{R}\left[\hat{\mathcal{L}}_{S R}(t) \hat{\mathcal{G}}_{P}\left(t, t_{0} \mid g\right)(1-\mathcal{P}) W\right]$. (4) In the second equality of Eq. (B1), $e^{-\mathcal{L}_{0}(t-\tau)}$ and $e^{\mathcal{L}_{0}(t-\tau)}$ should be replaced by $e^{-\mathcal{L}_{0}\left(t-t_{0}\right)}$ and $e^{\mathcal{L}_{0}\left(\tau-t_{0}\right)}$, respectively. 